\title{
Work fluctuation relation of an active Brownian particle in a viscoelastic fluid
}

\author{
N Narinder $\odot$, Shuvojit Paul $\odot$, and Clemens Bechinger $\odot^{*}$ \\ Fachbereich Physik, Universität Konstanz, 78464 Konstanz, Germany
}

(Received 9 June 2021; accepted 4 August 2021; published 7 September 2021)

\begin{abstract}
We experimentally investigate the work fluctuations of an active Brownian particle (ABP) during its selfpropelled motion in a viscoelastic medium. Under such conditions, ABPs display a persistent circular motion which allows the determination of the orientational work fluctuations along its trajectory. Due to the nonlinear coupling to the non-Markovian bath, we find strong deviations from the work fluctuation theorem (WFT) due to observed increased rotational ABP dynamics. Taking this enhanced rotational diffusion into account, the orientational work distributions can be recasted to be in accordance with the WFT by considering an effective temperature of about two orders of magnitude larger than $k_{\mathrm{B}} T$. This approach is confirmed by the good agreement of the torque exerted by the viscoelastic bath on the ABP obtained from the WFT with the value obtained from the mean angular velocity and the friction coefficient of the ABP.
\end{abstract}

DOI: 10.1103/PhysRevE.104.034605

\section{INTRODUCTION}

Active Brownian particles (ABPs) which self-propel through a fluid medium currently receive considerable attention as intrinsic nonequilibrium model systems [1-4]. Unlike Brownian particles, they dissipate heat into their surroundings and thus break detailed balance [5]. In purely viscous, i.e., Newtonian fluids, the ABPs' orientational dynamics is entirely determined by thermal fluctuations, which eventually leads to a persistent random walk with a crossover time from ballistic to diffusive motion independent of the propulsion velocity [6-9]. Quite unlike this, ABPs show a strong coupling of their translational and orientational dynamics when propelling in a viscoelastic fluid, which leads to, e.g., a dramatic enhancement of rotational diffusion [10-14] and even persistent circular trajectories [15]. Both phenomena originate from the strong nonlinear coupling of the ABP's orientation to the surrounding viscoelastic bath which is absent in viscous fluids.

Microscopic information regarding the coupling of a particle to its surroundings can be obtained using the work fluctuation theorem (WFT), which quantifies the distribution of work in microscopic systems [16,17]. According to the WFT, for a system in contact with a thermal bath at temperature $T$, driven to a nonequilibrium steady state

$$
\ln \frac{P\left(+W_{\tau}\right)}{P\left(-W_{\tau}\right)}=\frac{W_{\tau}}{k_{\mathrm{B}} T}, \quad \tau \gg \tau_{c},
$$

where $P\left(+W_{\tau}\right)$ and $P\left(-W_{\tau}\right)$ are the probabilities of positive and corresponding negative work $W_{\tau}$ in time $\tau, k_{\mathrm{B}}$ is the Boltzmann constant and $\tau_{c}$ represents the longest relaxation time of the system. Equation (1) has been experimentally verified in numerous experiments, for instance, during the folding and unfolding of RNA hairpins [18], the motion of trapped colloids in time-dependent potentials [19-22], and

\footnotetext{
*clemens.bechinger@uni-konstanz.de
}

even for electronic systems [23]. Notably, in all of these studies, an external driving force has been applied and only rare examples exist where an internal driving mechanism has been considered [24-26]. All mentioned examples, however, have been carried out in purely viscous media, providing a linear coupling to the probe. Given the fact that many biological media are viscoelastic, however, it is also interesting to study whether the WFT remains valid in case of a nonlinear coupling between the probe and the bath.

Here, we experimentally investigate the orientational work fluctuations of an ABP in a viscoelastic semidilute polymer network. We observe deviations by more than one order of magnitude from WFT, which are caused by the strong enhancement of the ABP's rotational diffusion. After introducing an effective temperature, the WFT can be largely restored, which allows the calculation of the torque exerted by the viscoelastic fluid on the ABP.

\section{EXPERIMENTAL DETAILS}

Our experiments have been performed in a solution of polyacrylamide $(\approx 0.05 \mathrm{wt} \%)$ dissolved in water. When adding propylene glycol $n$-propyl ether (PnP) with a mass ratio $2: 3$, we obtain a critical mixture with a lower demixing temperature at $T_{c}=304.6 \mathrm{~K}$ [10]. The temperature of the sample is kept at $T=296 \pm 0.01 \mathrm{~K}$ using a bath thermostat. Using passive microrheology [27], we determined the viscosity of the solvent to $\eta_{\infty}=0.13 \pm 0.02 \mathrm{~Pa}$ s and $\eta_{0}=0.72 \pm$ $0.08 \mathrm{~Pa} \mathrm{~s}$ without and with added polymer, respectively. The fluid's stress relaxation time $\tau_{0}$ has been determined via a recoil experiment to $1.52 \pm 0.40 \mathrm{~s}$ [27]. ABPs were made from silica spheres with radius $a=3.88 \mu \mathrm{m}$ which are capped from one side with a 50-nm carbon layer. We suspend the particles to the critical fluid and confine them in a cell of height $h \approx 4 a$. Under such conditions, the translational and rotational motion of the particles is limited to two dimensions [28]. We illuminate the sample cell with a green laser $(\lambda=532 \mathrm{~nm})$ which is partially absorbed by the carbon caps. 

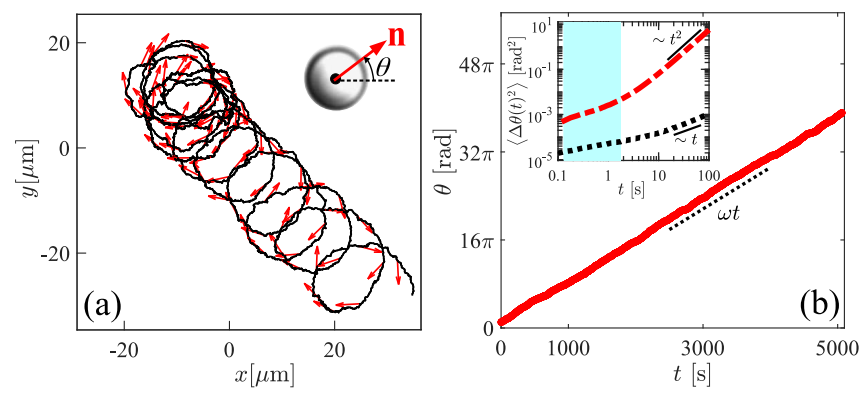

FIG. 1. (a) Typical trajectory of a spherical ABP in a viscoelastic fluid displaying a persistent circular motion with angular velocity $\omega=0.025 \mathrm{rad} \mathrm{s}^{-1}$. The red arrows denote the particle orientation. Inset: optical image of a single ABP with the dark side corresponding to the carbon coating. The particle orientation is defined by the angle between the vector $\mathbf{n}$, which points from capped to the uncapped side, and the $x$ axis. (b) Time dependence of the accumulated particle orientation showing a linear behavior $\omega t$. Inset: Angular mean squared displacements $\left\langle\Delta \theta(t)^{2}\right\rangle$ of an ABP (dash-dotted line) and of a Brownian particle (dotted line). Below the stress relaxation time $\tau_{0}$ the behavior becomes subdiffusive due to the elastic properties of the solvent.

This leads to a heating of the cap resulting in local demixing of the fluid and eventually to self-propulsion of the particles [29]. The ABP's propulsion speed $v$ can be controlled by the intensity of the illumination which has been varied between 10 and $20 \mu \mathrm{W} \mu \mathrm{m}^{-2}$. Particle positions $\mathbf{r}=(x, y)$ and their in-plane orientation $\mathbf{n}=(\cos \theta, \sin \theta)$ were measured using optical microscopy and tracked using a standard image processing software [30].

\section{ORIENTATIONAL WORK FLUCTUATIONS OF AN ACTIVE PARTICLE UNDERGOING PERSISTENT CIRCULAR MOTION}

As reported previously [15], ABPs propelling through viscoelastic fluids exhibit a persistent circular motion when
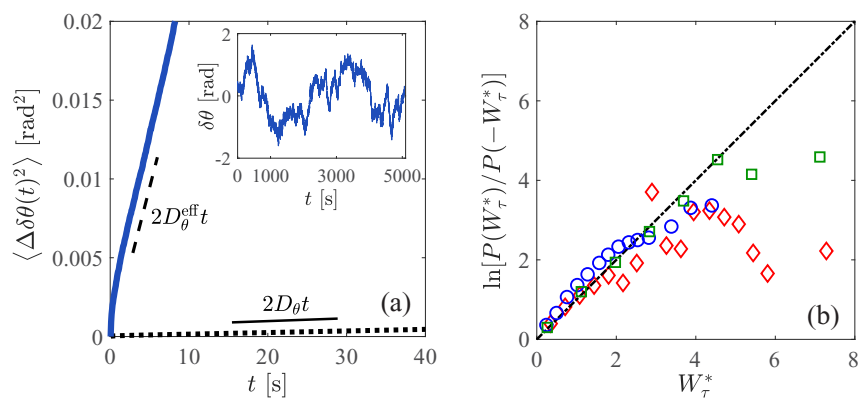

FIG. 3. (a) The angular mean squared displacement $\left\langle\delta \Delta \theta(t)^{2}\right\rangle$ of the angular fluctuations $\delta \theta$ (solid curve) obtained by subtracting the angular drift $\omega t$ from the time evolution of angular coordinate $\theta$ for an ABP rotating with an angular velocity $\omega=0.025 \mathrm{rad} \mathrm{s}^{-1}$ and the corresponding angular mean squared displacement of a Brownian particle in the same viscoelastic fluid (dotted curve). Inset: The time evolution of the angular fluctuations $\delta \theta$ around the mean circular path for the ABP rotating with an angular velocity $\omega=0.025 \mathrm{rad} \mathrm{s}^{-1}$. (b) $\ln \left[P\left(+W_{\tau}^{*}\right) / P\left(-W_{\tau}^{*}\right)\right]$ vs $W_{\tau}^{*}$ of the PDF of the normalized work $W_{\tau}^{*}$ for different angular velocities: $\omega=0.018 \mathrm{rad} \mathrm{s}^{-1}$ (diamonds), $0.025 \mathrm{rad} \mathrm{s}^{-1}$ (circles), and $0.095 \mathrm{rad} \mathrm{s}^{-1}$ (squares). The dash-dotted line corresponds to the work fluctuation theorem.

their propulsion velocity is increased above a critical value [Fig. 1(a)]. Such behavior is explained by a time-delayed fluid's response to the particle motion which leads to a misalignment between the propulsion force and the ABP's orientation, which eventually generates a constant and velocity-dependent torque exerted by the fluid on the ABP. The circular motion is confirmed by the linear increase of the accumulated angular coordinate $\theta$, which is plotted in Fig. 1(b). Accordingly, the angular mean-squared displacement $\left\langle\Delta \theta(t)^{2}\right\rangle$ exhibits a ballistic behavior at time scales above the stress relaxation time $\tau_{0}$ [inset Fig. 1(b)]. Below $\tau_{0}$, the particle motion is subdiffusive being a typical signature of particles coupled to viscoelastic media such as polymers and dense colloidal suspensions, etc. [31-34].
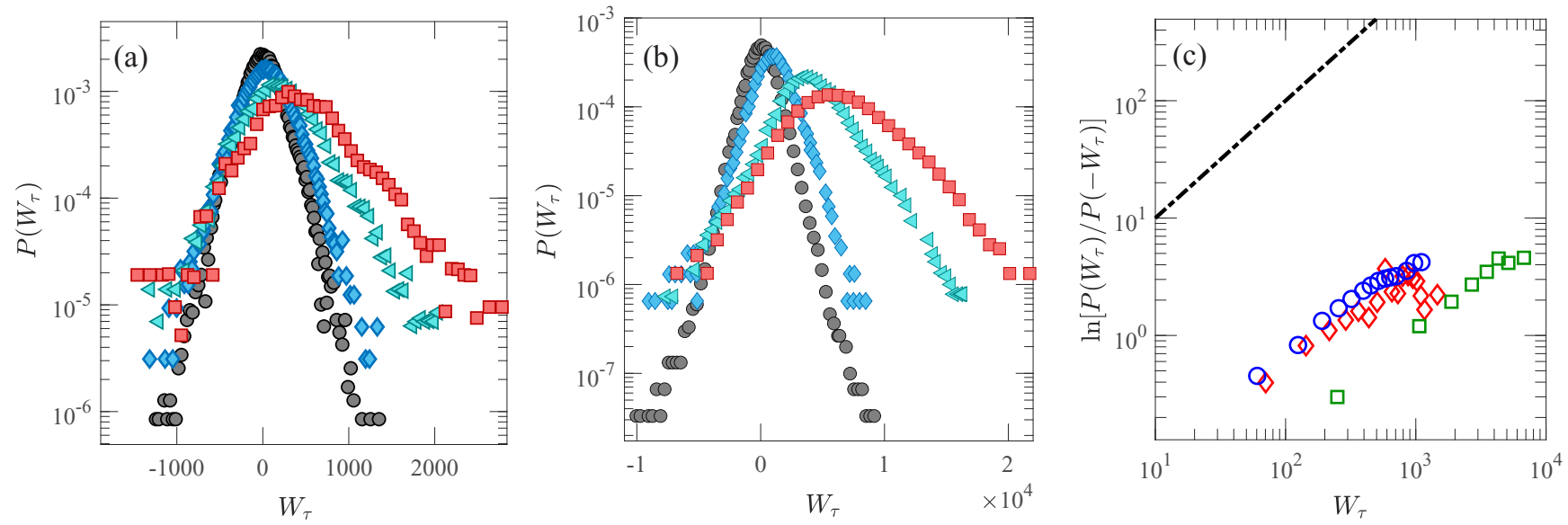

FIG. 2. Probability density functions of the work $W_{\tau}$ for spherical active particles moving in viscoelastic fluid with different angular velocities (a) $\omega=0.018 \mathrm{rad} \mathrm{s}^{-1}$ and (b) $\omega=0.095 \mathrm{rad} \mathrm{s}^{-1}$. The symbols correspond to the integration times $\tau=0.06 \mathrm{~s}$ (circles), $0.59 \mathrm{~s}$ (diamonds), $2.94 \mathrm{~s}$ (triangles), and $5.88 \mathrm{~s}$ (squares). (c) $\ln \left[P\left(+W_{\tau}\right) / P\left(-W_{\tau}\right)\right]$ as a function of $W_{\tau}$ computed for integration time $\tau=5.88 \mathrm{~s}$ for the active particles moving in circles in the viscoelastic fluid at angular velocities $\omega=0.018 \mathrm{rad} \mathrm{s}^{-1}$ (diamonds), $0.025 \mathrm{rad} \mathrm{s}{ }^{-1}$ (circles), and $0.095 \mathrm{rad} \mathrm{s}^{-1}$ (squares). The dash-dotted line corresponds to the work fluctuation theorem. 

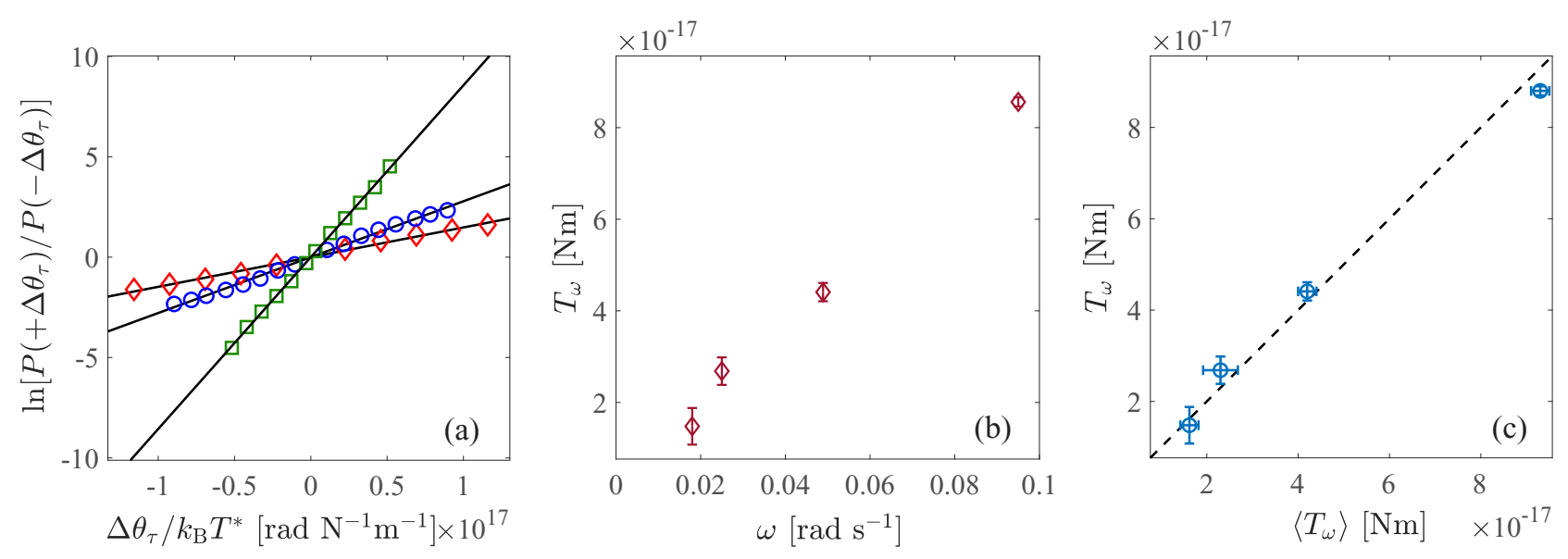

FIG. 4. (a) $\ln \left[P\left(\Delta \theta_{\tau}\right) / P\left(-\Delta \theta_{\tau}\right)\right]$ of the PDFs of angular change $\Delta \theta_{\tau} / k_{\mathrm{B}} T^{*}$ for the ABP in the viscoelastic fluid, calculated for the integration time $\tau=5.88 \mathrm{~s}$ for different angular velocities $\omega=0.018 \mathrm{rad} \mathrm{s}^{-1}$ (diamonds), $0.025 \mathrm{rad} \mathrm{s}^{-1}$ (circles), and $0.095 \mathrm{rad} \mathrm{s}^{-1}$ (squares). The solid lines are the linear fits to the corresponding curves. (b) The measured torque acting on spherical Janus particles using the normalized fluctuation relation as a function of angular velocity $\omega$. (c) Comparison of torques measured using fluctuation relation $T_{\omega}$ with the mean estimated values $\left\langle T_{\omega}\right\rangle$ computed using Eq. (3). The dashed line corresponds to line with slope unity.

In the presence of a constant torque which leads to a circular mean particle trajectory, the orientational work $W_{\tau}$ during time $\tau$ done by an ABP is given by

$$
W_{\tau}=\frac{1}{k_{B} T} \int_{t}^{t+\tau} \dot{\theta}\left(t^{\prime}\right)\left\langle T_{\omega}\right\rangle d t^{\prime},
$$

where $\left\langle T_{\omega}\right\rangle$ is the mean torque required to rotate a sphere with radius $a$ at angular velocity $\omega$ in a medium with viscosity $\eta(\omega)$

$$
\left\langle T_{\omega}\right\rangle=8 \pi a^{3} \eta(\omega) \omega
$$

The viscosity $\eta(\omega)$ has been obtained from the mean squared displacement of a Brownian particle embedded in the viscoelastic fluid [27]. Accordingly, the measurement of $\dot{\theta}(t)$ provides a direct computation of $W_{\tau}$. The work done is assumed to be positive if a change in angular coordinate after a time interval $\tau$ with regard to previous orientation; i.e., $\Delta \theta=\theta(t+\tau)-\theta(t)$ occurs toward the direction of rotation and negative otherwise. The probability distributions (PDFs) $P\left(W_{\tau}\right)$ are plotted in Fig. 2 for different integration times $\tau$ and for $\omega=0.018$ and $0.095 \mathrm{rad} \mathrm{s}^{-1}$. The maxima of the PDFs become increasingly shifted away from the center for larger $\tau$, reflecting the circular particle motion. In addition, we observe an asymmetry of the work distribution for larger $\tau$. This asymmetry results from the nonlinear coupling of the $\mathrm{ABP}$ to the viscoelastic fluid and is absent in case of a purely viscous bath [27]. Similar non-Gaussian PDFs have been also observed in externally driven nonlinear systems $[35,36]$. To test the validity of the WFT, in Fig. 2(c) we have plotted $\ln \left[P\left(+W_{\tau}\right) / P\left(-W_{\tau}\right)\right]$ versus $W_{\tau}$ in a $\log -\log$ representation for different values of $\omega$. Clearly, the curves deviate by more than one order of magnitude from Eq. (1) (dash-dotted line).

\section{RENORMALIZATION OF THE WORK BY THE EFFECTIVE TEMPERATURE}

In the following, we want to understand the origin of the deviations from the WFT in more detail. For this purpose, we consider the rotational diffusive dynamics of the $\mathrm{ABP}$ while describing a circular motion. This is done by removing the angular drift $\omega t$ from the time evolution of $\theta(t)$ which corresponds to the fluctuations around the mean circular path, i.e., $\delta \theta(t)=\theta(t)-\omega t+$ const. as shown in the inset of Fig. 3(a). The mean squared displacement of the angular fluctuations exhibit a diffusive behavior, i.e., $\Delta \delta \theta(t)^{2}=2 D_{\theta}^{\text {eff }} t$ for $t \gtrsim \tau_{0}$ with $D_{\theta}^{\text {eff }}$ being enhanced by two orders of magnitude compared to a free Brownian particle [Fig. 3(a)]. For a qualitative understanding of this enhancement, one has to consider that the ABP induces a local stress in the viscoelastic polymer network which relaxes over time $\sim \tau_{0}$. This couples back to the ABPs stochastic orientational motion, enhancing the angular fluctuations and thereby increasing its rotational diffusion coefficient. Such an enhancement can be formally expressed by introducing an effective temperature $T^{*}=\frac{D_{\theta}^{\text {eff }}}{D_{\theta}} T$. For the example shown in Fig. 3(a), $T^{*} \approx 7.4 \times 10^{4} \mathrm{~K}$. Because the rotational dynamics of the $\mathrm{ABP}$ on its circular trajectory is governed by a higher effective temperature, this must be considered for the normalization of the work $W_{\tau}$. This leads to [37]

$$
W_{\tau}^{*}=\frac{W_{\tau}}{\alpha},
$$

where $\alpha=D_{\theta}^{\text {eff }} / D_{\theta}$. To test whether Eq. (1) remains valid when considering an effective temperature, we have replotted the data in Fig. 2(c) but now with replacing $W_{\tau}$ by $W_{\tau}^{*}$ [Fig. 3(b)]. Compared to Fig. 2(c), the agreement with the WFT (dash-dotted line) is strongly enhanced, in particular at values $W_{\tau}^{*} \lesssim 3$. For larger values of work, i.e., $W_{\tau}^{*} \gtrsim 3$, the deviations from the linear behavior arise due to rare but rather extreme angular fluctuations in response to the coupling to the viscoelastic bath. Such deviations have been also observed in other systems when the variance of the fluctuating quantity becomes considerably larger than the thermal fluctuations $[37,38]$.

As a test confirming the validity of our approach, we finally calculate the torque acting on the ABP using the renormalized WFT. Utilizing Eq. (1) for the range $W_{\tau}^{*} \lesssim 3$ where our data 
agree with the WFT, $W_{\tau}^{*}=T_{\omega} \Delta \theta_{\tau} / k_{\mathrm{B}} T^{*}$, provides a direct measure of the torque. The result is shown in Fig. 4(a) for different $\omega$. The measured torque $T_{\omega}$ as a function of $\omega$ is plotted in Fig. 4(b). Comparison of the results with the corresponding estimated mean value using Eq. (3) shows excellent agreement and a posteriori justifies our approach [Fig. 4(c)].

\section{SUMMARY AND CONCLUSIONS}

In summary, we have studied the orientational work fluctuations during the memory-induced persistent circular motion of an active particle in a viscoelastic fluid. We find strong deviations from the WFT which are explained by the nonlinear coupling of the ABP to the non-Markovian bath, resulting in a strongly enhanced rotational diffusion. Taking this effect into account, we are able to recast the WFT by introducing an effective temperature. The validity of our approach is demonstrated by the excellent agreement of the torque exerted on the ABP based on the re-established WFT and the corresponding value directly obtained from the angular particle velocity during its circular persistent motion. This suggests the use of fluctuation theorems to provide important information of active particles without the need for their friction coefficients and irrespective of their geometrical shape.

\section{ACKNOWLEDGMENTS}

The authors acknowledge stimulating discussions with Thomas Speck. This work is financially supported by the Alexander von Humboldt Foundation (S.P.) and the European Commission through the ERC Grant No. 693683 ASCIR.
[1] C. Bechinger, R. Di Leonardo, H. Löwen, C. Reichhardt, G. Volpe, and G. Volpe, Active particles in complex and crowded environments, Rev. Mod. Phys. 88, 045006 (2016).

[2] W. Wang, L. A. Castro, M. Hoyos, and T. E. Mallouk, Autonomous motion of metallic microrods propelled by ultrasound, ACS Nano 6, 6122 (2012).

[3] L. Baraban, R. Streubel, D. Makarov, L. Han, D. Karnaushenko, O. G. Schmidt, and G. Cuniberti, Fuel-free locomotion of janus motors: Magnetically induced thermophoresis, ACS Nano 7, 1360 (2013).

[4] B. Dai, J. Wang, Z. Xiong, X. Zhan, W. Dai, C.-C. Li, S.-P. Feng, and J. Tang, Programmable artificial phototactic microswimmer, Nat. Nanotechnol. 11, 1087 (2016).

[5] T. Speck, Active Brownian particles driven by constant affinity, EPL 123, 20007 (2018).

[6] J. Palacci, C. Cottin-Bizonne, C. Ybert, and L. Bocquet, Sedimentation and Effective Temperature of Active Colloidal Suspensions, Phys. Rev. Lett. 105, 088304 (2010).

[7] H.-R. Jiang, N. Yoshinaga, and M. Sano, Active Motion of a Janus Particle by Self-Thermophoresis in a Defocused Laser Beam, Phys. Rev. Lett. 105, 268302 (2010).

[8] J. R. Howse, R. A. L. Jones, A. J. Ryan, T. Gough, R. Vafabakhsh, and R. Golestanian, Self-Motile Colloidal Particles: From Directed Propulsion to Random Walk, Phys. Rev. Lett. 99, 048102 (2007).

[9] I. Buttinoni, G. Volpe, F. Kümmel, G. Volpe, and C. Bechinger, Active Brownian motion tunable by light, J. Phys.: Condens. Matter 24, 284129 (2012).

[10] J. R. Gomez-Solano, A. Blokhuis, and C. Bechinger, Dynamics of Self-Propelled Janus Particles in Viscoelastic Fluids, Phys. Rev. Lett. 116, 138301 (2016).

[11] C. Lozano, J. R. Gomez-Solano, and C. Bechinger, Active particles sense micromechanical properties of glasses, Nat. Mater. 18, 1118 (2019).

[12] K. Qi, E. Westphal, G. Gompper, and R. G. Winkler, Enhanced Rotational Motion of Spherical Squirmer in Polymer Solutions, Phys. Rev. Lett. 124, 068001 (2020).

[13] N. Narinder, J. R. Gomez-Solano, and C. Bechinger, Active particles in geometrically confined viscoelastic fluids, New J. Phys. 21, 093058 (2019).
[14] C. Abaurrea-Velasco, C. Lozano, C. Bechinger, and J. de Graaf, Autonomously Probing Viscoelasticity in Disordered Suspensions, Phys. Rev. Lett. 125, 258002 (2020).

[15] N. Narinder, C. Bechinger, and J. R. Gomez-Solano, MemoryInduced Transition from a Persistent Random Walk to Circular Motion for Achiral Microswimmers, Phys. Rev. Lett. 121, 078003 (2018).

[16] D. J. Evans, E. G. D. Cohen, and G. P. Morriss, Probability of Second Law Violations in Shearing Steady States, Phys. Rev. Lett. 71, 2401 (1993).

[17] G. E. Crooks, Entropy production fluctuation theorem and the nonequilibrium work relation for free energy differences, Phys. Rev. E 60, 2721 (1999).

[18] D. Collin, F. Ritort, C. Jarzynski, S. B. Smith, I. Tinoco, and C. Bustamante, Verification of the Crooks fluctuation theorem and recovery of RNA folding free energies, Nature (London) 437, 231 (2005).

[19] F. Douarche, S. Joubaud, N. B. Garnier, A. Petrosyan, and S. Ciliberto, Work Fluctuation Theorems for Harmonic Oscillators, Phys. Rev. Lett. 97, 140603 (2006).

[20] G. M. Wang, E. M. Sevick, E. Mittag, D. J. Searles, and D. J. Evans, Experimental Demonstration of Violations of the Second Law of Thermodynamics for Small Systems and Short Time Scales, Phys. Rev. Lett. 89, 050601 (2002).

[21] A. Imparato, L. Peliti, G. Pesce, G. Rusciano, and A. Sasso, Work and heat probability distribution of an optically driven Brownian particle: Theory and experiments, Phys. Rev. E 76, 050101(R) (2007).

[22] A. Imparato, P. Jop, A. Petrosyan, and S. Ciliberto, Probability density functions of work and heat near the stochastic resonance of a colloidal particle, J. Stat. Mech.: Theory Exp. (2008) P10017.

[23] O.-P. Saira, Y. Yoon, T. Tanttu, M. Möttönen, D. V. Averin, and J. P. Pekola, Test of the Jarzynski and Crooks Fluctuation Relations in an Electronic System, Phys. Rev. Lett. 109, 180601 (2012).

[24] K. Hayashi, H. Ueno, R. Iino, and H. Noji, Fluctuation Theorem Applied to $F_{1}$-Atpase, Phys. Rev. Lett. 104, 218103 (2010).

[25] T. Speck, Stochastic thermodynamics for active matter, EPL 114, 30006 (2016). 
[26] S. Chaki and R. Chakrabarti, Entropy production and work fluctuation relations for a single particle in active bath, Physica A (Amsterdam, Neth.) 511, 302 (2018).

[27] See Supplemental Material at http://link.aps.org/supplemental/ 10.1103/PhysRevE.104.034605 for the detailed calculationsfor determining the rheological parameters of the viscoelastic fluid and the work fluctuation theorem applied to L-shaped particles in the counterpart Newtonian fluid, which includes Refs. [3942].

[28] S. Das, A. Garg, A. I. Campbell, J. Howse, A. Sen, D. Velegol, R. Golestanian, and S. J. Ebbens, Boundaries can steer active Janus spheres, Nat. Commun. 6, 8999 (2015).

[29] J. R. Gomez-Solano, S. Samin, C. Lozano, P. Ruedas-Batuecas, R. van Roij, and C. Bechinger, Tuning the motility and directionality of self-propelled colloids, Sci. Rep. 7, 14891 (2017).

[30] J. C. Crocker and D. G. Grier, Methods of digital video microscopy for colloidal studies, J. Colloid Interface Sci. 179, 298 (1996).

[31] E. Andablo-Reyes, P. Díaz-Leyva, and J. L. Arauz-Lara, Microrheology from Rotational Diffusion of Colloidal Particles, Phys. Rev. Lett. 94, 106001 (2005).

[32] Z. Cheng and T. G. Mason, Rotational Diffusion Microrheology, Phys. Rev. Lett. 90, 018304 (2003).

[33] E. R. Weeks and D. A. Weitz, Subdiffusion and the cage effect studied near the colloidal glass transition, Chem. Phys. 284, 361 (2002).

[34] R. B. Bird, R. C. Armstrong, and O. Hassager, Dynamics of Polymeric Liquids (Fluid Mechanics), Vol. 1 (John Wiley and Sons, New York, 1987).
[35] M. Bonaldi, L. Conti, P. De Gregorio, L. Rondoni, G. Vedovato, A. Vinante, M. Bignotto, M. Cerdonio, P. Falferi, N. Liguori et al., Nonequilibrium Steady-State Fluctuations in Actively Cooled Resonators, Phys. Rev. Lett. 103, 010601 (2009).

[36] E. Falcon, S. Aumaître, C. Falcón, C. Laroche, and S. Fauve, Fluctuations of Energy Flux in Wave Turbulence, Phys. Rev. Lett. 100, 064503 (2008).

[37] J. R. Gomez-Solano, L. Bellon, A. Petrosyan, and S. Ciliberto, Steady-state fluctuation relations for systems driven by an external random force, EPL 89, 60003 (2010).

[38] C. Falcón and E. Falcon, Fluctuations of energy flux in a simple dissipative out-of-equilibrium system, Phys. Rev. E 79, 041110 (2009).

[39] J. R. Gomez-Solano and C. Bechinger, Transient dynamics of a colloidal particle driven through a viscoelastic fluid, New J. Phys. 17, 103032 (2015).

[40] S. Paul, N. Narinder, A. Banerjee, K. R. Nayak, J. Steindl, and C. Bechinger, Bayesian inference of the viscoelastic properties of a Jeffreys fluid using optical tweezers, Sci. Rep. 11, 2023 (2021).

[41] T. G. Mason and D. A. Weitz, Optical Measurements of Frequency-Dependent Linear Viscoelastic Moduli of Complex Fluids, Phys. Rev. Lett. 74, 1250 (1995).

[42] F. Kümmel, B. ten Hagen, R. Wittkowski, I. Buttinoni, R. Eichhorn, G. Volpe, H. Löwen, and C. Bechinger, Circular Motion of Asymmetric Self-Propelling Particles, Phys. Rev. Lett. 110, 198302 (2013). 